\title{
Full Wave Modeling of Array Structures Using Generalized Single-Source Tangential Equivalence Principle Algorithm
}

\author{
Hanru Shao, ${ }^{1}$ Jianfeng Dong, ${ }^{1}$ and Jun $\mathrm{Hu}^{2}$ \\ ${ }^{1}$ Faculty of Electrical Engineering and Computer Science, Ningbo University, Ningbo 315211, China \\ ${ }^{2}$ School of Electronic Engineering, University of Electronic Science and Technology of China, Chengdu 611731, China \\ Correspondence should be addressed to Hanru Shao; shaohanru@nbu.edu.cn
}

Received 28 April 2017; Accepted 28 June 2017; Published 6 August 2017

Academic Editor: Yumao Wu

Copyright (c) 2017 Hanru Shao et al. This is an open access article distributed under the Creative Commons Attribution License, which permits unrestricted use, distribution, and reproduction in any medium, provided the original work is properly cited.

\begin{abstract}
The generalized single-source tangential equivalence principle algorithm (GSST-EPA) is proposed to solve the electromagnetic scattering of array structures with very small distance or even connected elements. For the traditional EPA scheme, it is difficult to deal with those situations. Based on source reconstruction method (SRM), the new GSST-EPA can be used to solve this problem efficiently. This scheme can also reduce the number of unknowns by half via the extinction theorem and keep the good accuracy by using tangential field projection. Furthermore, the multilevel fast multipole algorithm (MLFMA) is used to accelerate the matrixvector multiplication in the GSST-EPA. Several numerical results are given to demonstrate the accuracy and efficiency of the proposed method.
\end{abstract}

\section{Introduction}

The array structure has a great range of applications in practical engineering, such as antenna arrays, metamaterials, and microwave absorber. As a kind of multiscale modeling, the array structure involving large number of elements and fine components has been the challenge for traditional full wave methods [1]. Therefore, more advanced numerical methods with better efficiency and robustness need to be developed.

In recent years, many efforts have been made to address the above problems. Particularly, the full wave integral equation (IE) solvers have attracted much attention due to high accuracy, relatively small matrices, and ability to be accelerated by fast algorithms. The progress in IE solvers for array structures can be divided into two categories: one is macro basis function methods focusing on reducing the number of unknowns, for instance, the sub-entire-domain (SED) basis function method [2], the characteristic basis function (CBF) method [3], and the synthetic basis function (SBF) approach [4]. These methods are similar to each other except the way of generating macro basis function. The other one is domain decomposition methods based on integral equations, including equivalence principle algorithm (EPA) [5-7], linear embedding via Green's operator (LEGO) [8], and generalized transition matrix (GTM) [9]. The objectives of these methods are similar by using surface equivalence theorem to transform the unknowns on original objects onto the equivalence surfaces enclosing the objects.

The EPA has been used to solve some multiscale problems. Several efforts have also been made to improve the accuracy and extend the application range. For example, the highorder field point sampling scheme [7] and tangential EPA (TEPA) [10] have been proposed to improve the accuracy of scattering operator. The efficiency of scattering operator has been improved by hierarchical LU decomposition method [11]. Furthermore, the low-frequency problem [12] and timedomain models [13] have been solved using EPA method. The EPA is also combined with BOR to solve large-scale problems [14].

In this paper, the EPA is implemented to solve largescale array structures and some important improvements are made to achieve better numerical flexibility and efficiency. Firstly, the single-source EPA is used to reduce the number of unknowns by half via extinction theorem, in which only electric currents or magnetic currents on the equivalence surfaces need to be solved [15]. Secondly, the tangential field projection scheme is applied on equivalence surface to improve the accuracy of single-source EPA, which is 


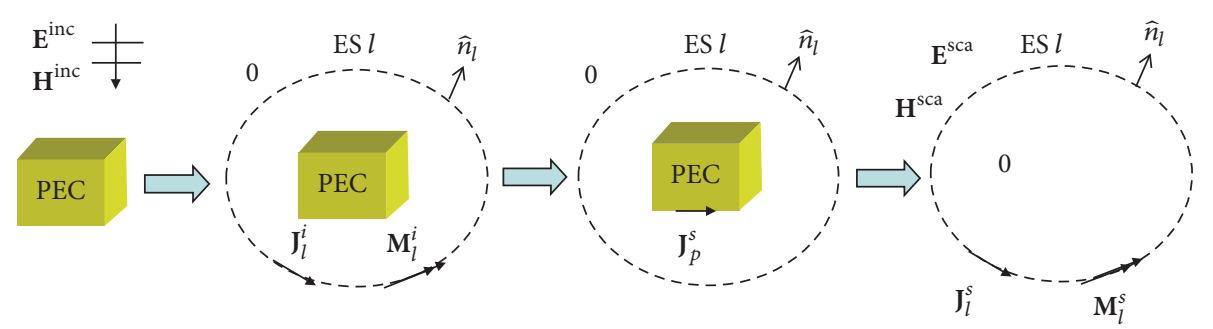

FIGURE 1: A PEC object characterized by equivalence surface with three steps.

called single-source tangential EPA (T-EPA) [16]. Thirdly, the source reconstruction method (SRM) is proposed to solve the case that connected structures intercepted by equivalence surfaces [17]. Using this scheme, the EPA equations are still well-conditioned and only equivalence surface currents are the final unknowns to be solved. Finally, the above three improvements are combined together as a new scheme named generalized single-source tangential EPA (GSSTEPA). The GSST-EPA is more efficient and robust than traditional EPA for solving complicated array problems. To improve the ability of solving large-scale array structures further, the multilevel fast multipole algorithm (MLFMA) [18] is applied to accelerate the translation procedure which is the coupling between nonadjacent equivalence surfaces. Numerical results are shown to validate the accuracy and efficiency of the new method.

\section{Equivalence Principle Algorithm}

The basic idea of EPA is to transform the original problems into a new equivalent problem with scattering operator and translation operator. The electromagnetic scattering of $N$ nonconnected perfect electric conductor (PEC) objects in free space is considered to illustrate EPA. Each object is enclosed by an artificial equivalence surface ES $l(l=$ $1, \ldots, N)$. The EPA scheme is based on the following two procedures: the scattering solution of objects via an equivalence surface and the couplings between objects via the equivalence surfaces, corresponding to the scattering operator and translation operator separately. Next, these two operators are defined in detail.

2.1. Scattering Operator. The first procedure can be derived from a PEC object characterized by equivalence surface with three steps as shown in Figure 1. The first step is outside-in propagation. The equivalence incident electric and magnetic currents on the equivalence surface can be obtained by using equivalence principle as follows:

$$
\begin{aligned}
\mathbf{J}_{l}^{i} & =-\widehat{n}_{l} \times \mathbf{H}^{\mathrm{inc}}, \\
\mathbf{M}_{l}^{i} & =\widehat{n}_{l} \times \mathbf{E}^{\mathrm{inc}},
\end{aligned}
$$

where $\widehat{n}_{l}$ is the unit outer normal vector on the ES $l$. The currents $\mathbf{J}_{l}^{i}$ and $\mathbf{M}_{l}^{i}$ generate the original incident fields inside and zero field outside. Then the electric current on the PEC can be solved using method of moments (MoM) which is the second step as

$$
\eta_{0} \mathscr{L}\left(\mathbf{J}_{p}^{s}\right)=\left[\begin{array}{lll}
-\eta_{0} \mathscr{L}_{p l} & \eta_{0} \mathscr{K}_{p l}
\end{array}\right] \cdot\left[\begin{array}{c}
\mathbf{J}_{l}^{i} \\
\frac{1}{\eta_{0}} \mathbf{M}_{l}^{i}
\end{array}\right] .
$$

Here, $\mathscr{L}$ and $\mathscr{K}$ are the surface integral operators defined as

$$
\begin{aligned}
\mathscr{L}\left(\mathbf{r}, \mathbf{r}^{\prime}\right) \cdot \mathbf{X}\left(\mathbf{r}^{\prime}\right)= & i k \int_{s}\left[\mathbf{X}\left(\mathbf{r}^{\prime}\right)+\frac{1}{k^{2}} \nabla \nabla^{\prime} \cdot \mathbf{X}\left(\mathbf{r}^{\prime}\right)\right] \\
& \cdot G_{0}\left(\mathbf{r}, \mathbf{r}^{\prime}\right) d s^{\prime}, \\
\mathscr{K}\left(\mathbf{r}, \mathbf{r}^{\prime}\right) \cdot \mathbf{X}\left(\mathbf{r}^{\prime}\right)= & \text { P.V. } \int_{s} \nabla G_{0}\left(\mathbf{r}, \mathbf{r}^{\prime}\right) \times \mathbf{X}\left(\mathbf{r}^{\prime}\right) d s^{\prime},
\end{aligned}
$$

where $\mathbf{X}$ is $\mathbf{J}$ or $\mathbf{M} ; G_{0}$ is Green's function in free space. $\eta_{0}$ is the wave impedance in free space. P.V. stands for the Cauchy principle value integration. The last step is inside-out propagation. Once the PEC current is solved, the scattering electric and magnetic currents can be computed using equivalence principle as

$$
\begin{aligned}
& {\left[\begin{array}{cc}
\mathscr{I}_{l l} & 0 \\
0 & \mathscr{I}_{l l}
\end{array}\right] \cdot\left[\begin{array}{c}
\mathbf{J}_{l}^{s} \\
\frac{1}{\eta_{0}} \mathbf{M}_{l}^{s}
\end{array}\right]=\left[\begin{array}{c}
\widehat{n}_{l} \times \mathbf{H}^{\mathrm{sca}} \\
-\widehat{n}_{l} \times \mathbf{E}^{\mathrm{sca}}
\end{array}\right]} \\
& =\left[\begin{array}{c}
\widehat{n}_{l} \times \mathscr{K}_{l p} \\
\mathscr{L}_{l p} \times \widehat{n}_{l}
\end{array}\right] \cdot\left[\mathbf{J}_{p}^{s}\right]
\end{aligned}
$$

where $\mathscr{I}$ is identity operator. The currents $\mathbf{J}_{l}^{s}$ and $\mathbf{M}_{l}^{s}$ generate the original scattering fields outside and zero field inside. Combining these three steps, the scattering of a PEC object via an equivalence surface can be characterized by the scattering operator $\mathcal{S}_{l l}$ as

$$
\begin{aligned}
{\left[\mathbf{C}_{l}^{s}\right]=} & {\left[\begin{array}{cc}
\mathscr{I}_{l l} & 0 \\
0 & \mathscr{I}_{l l}
\end{array}\right]^{-1} \cdot\left[\begin{array}{c}
\widehat{n}_{l} \times \mathscr{K}_{l p} \\
\mathscr{L}_{l p} \times \widehat{n}_{l}
\end{array}\right] \cdot\left[\eta_{0} \mathscr{L}_{p p}\right]^{-1} } \\
& \cdot\left[-\eta_{0} \mathscr{L}_{p l} \quad \eta_{0} \mathscr{K}_{p l}\right] \cdot\left[\mathbf{C}_{l}^{i}\right]=\delta_{l l} \cdot\left[\mathbf{C}_{l}^{i}\right],
\end{aligned}
$$

where

$$
\left[\mathbf{C}_{l}^{s / i}\right]=\left[\begin{array}{c}
\mathbf{J}_{l}^{s / i} \\
\frac{1}{\eta_{0}} \mathbf{M}_{l}^{s / i}
\end{array}\right]
$$


It can be seen that the scattering operator can capture the full physics rigorously. Therefore, the unknowns on the inside object are transferred to the unknowns on the equivalence surface. Because the inside objects always have complex structures and different materials, the number of unknowns on the equivalence surface can be much less than that on the object.

2.2. Translation Operator. The second procedure can be illustrated by the scattering of two PEC objects as shown in Figure 2 . The field scattered by $\mathbf{J}_{k}^{s}$ and $\mathbf{M}_{k}^{s}$ produces new fields on ES $l$. So these fields can be interpreted as the incident currents from the ES $k$ to ES $l$ and can be expressed by a translation operator $\mathscr{T}_{l k}, l \neq k$, as

$$
\left[\mathbf{C}_{l k}^{i}\right]=\mathscr{T}_{l k} \cdot\left[\mathbf{C}_{k}^{s}\right]
$$

where

$$
\mathscr{T}_{l k}=\left[\begin{array}{cc}
\mathscr{I}_{l l} & 0 \\
0 & \mathscr{I}_{l l}
\end{array}\right]^{-1} \cdot\left[\begin{array}{cc}
-\widehat{n}_{l} \times \mathscr{K}_{l k} & -\widehat{n}_{l} \times \mathscr{L}_{l k} \\
\eta_{0} \widehat{n}_{l} \times \mathscr{L}_{l k} & -\eta_{0} \widehat{n}_{l} \times \mathscr{K}_{l k}
\end{array}\right] .
$$

Therefore, the scattered currents on ES $l$ can be written as

$$
\left[\mathbf{C}_{l}^{s}\right]=\mathcal{S}_{l l} \cdot\left[\mathbf{C}_{l}^{i}\right]+\mathscr{T}_{l k} \cdot\left[\mathbf{C}_{k}^{s}\right]
$$

For the generalized scattering of $N$ nonadjacent PEC objects enclosed by $N$ equivalence surfaces separately, the EPA equations can be written as

$$
\begin{aligned}
& {\left[\begin{array}{cccc}
\mathscr{I} & -\mathcal{S}_{11} \mathscr{T}_{12} & \cdots & -\mathcal{S}_{11} \mathscr{T}_{1 N} \\
-\mathcal{S}_{22} \mathscr{T}_{21} & \mathscr{I} & \cdots & -\mathcal{S}_{22} \mathscr{T}_{2 N} \\
\vdots & \vdots & \ddots & \vdots \\
-\mathcal{S}_{N N} \mathscr{T}_{N 1} & -\mathcal{S}_{N N} \mathscr{T}_{N 2} & \cdots & \mathscr{I}
\end{array}\right] \cdot\left[\begin{array}{c}
\mathbf{C}_{1}^{s} \\
\mathbf{C}_{2}^{s} \\
\vdots \\
\mathbf{C}_{N}^{s}
\end{array}\right]} \\
& =\left[\begin{array}{c}
\mathcal{S}_{11} \cdot \mathbf{C}_{1}^{i} \\
\mathcal{S}_{22} \cdot \mathbf{C}_{2}^{i} \\
\vdots \\
\mathcal{S}_{N N} \cdot \mathbf{C}_{N}^{i}
\end{array}\right] .
\end{aligned}
$$

The matrix form can be obtained by expanding the currents with Rao-Wilton-Glisson (RWG) basis function and using Galerkin's method to discretize the equation [19]. If the array structures have the same element, then each element can be enclosed by the same equivalence surface which leads to the same scattering operator. Therefore, only one scattering operator needs to be solved and stored which can reduce the solving time significantly.

\section{Single-Source Equivalence Principle Algorithm}

The single-source EPA is similar to original EPA scheme which is based on the above two procedures. The difference is that the equivalence magnetic currents on ES $l$ can be expressed by the equivalence electric currents on ES $l$ in

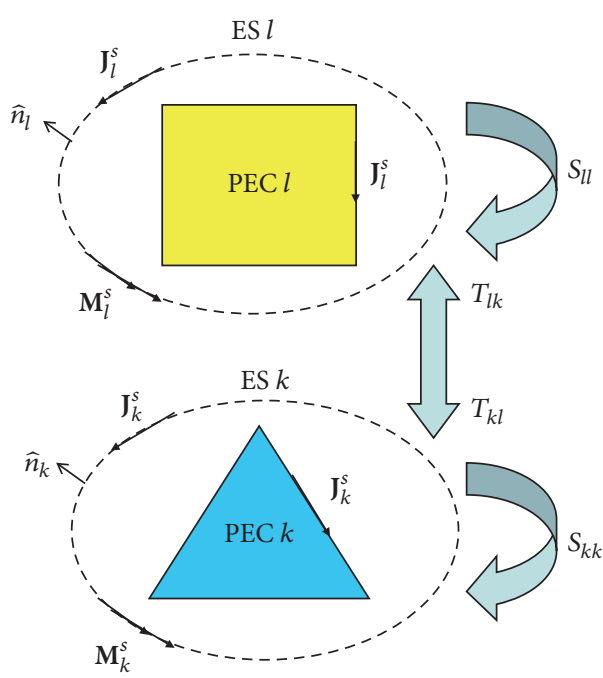

FIgUre 2: The interaction of two PEC objects using EPA scheme.

the single-source EPA. Therefore, only the electric currents on the equivalence surfaces are the final unknowns, which can reduce the number of unknowns by half compared with original EPA.

According to extinction theorem, the equivalence incident currents $\mathbf{J}_{l}^{i}$ and $\mathbf{M}_{l}^{i}$ generate zero field outside the ES $l$ as shown in Figure 3(a):

$$
\begin{aligned}
\mathbf{E}^{i}=\eta_{0} \mathscr{L}_{l l}\left(\mathbf{J}_{l}^{i}\right)+\frac{1}{2} \mathscr{J}_{l l}^{r}\left(\mathbf{M}_{l}^{i}\right)-\mathscr{K}_{l l}\left(\mathbf{M}_{l}^{i}\right)= & 0, \\
& \text { on } \mathrm{ES} l^{+}, \\
\mathbf{H}^{i}=-\frac{1}{2} \mathscr{I}_{l l}^{r}\left(\mathbf{J}_{l}^{i}\right)+\mathscr{K}_{l l}\left(\mathbf{J}_{l}^{i}\right)+\frac{1}{\eta_{0}} \mathscr{L}_{l l}\left(\mathbf{M}_{l}^{i}\right)= & 0, \\
& \text { on } \mathrm{ES} l^{+},
\end{aligned}
$$

where ES $l^{+}$denotes the outside surface of ES $l$. The integral operators $\mathscr{L}, \mathscr{K}$ are defined as (3), and $\mathscr{I}^{r}$ is defined as

$$
\mathscr{J}^{r}(\mathbf{X})=\widehat{n} \times \mathbf{X}
$$

$\mathbf{X}$ is equivalence incident or scattering surface current $\mathbf{J}^{i / s}$ or $\mathbf{M}^{i / s}$. Then the magnetic current can be expressed by electric current as

$$
\begin{array}{r}
\frac{1}{\eta_{0}} \mathbf{M}_{l}^{i}=\left[-\frac{1}{2} \mathscr{J}_{l l}^{r}+\mathscr{K}_{l l}\right]^{-1} \cdot \mathscr{L}_{l l}\left(\mathbf{J}_{l}^{i}\right)=\mathscr{Z}_{E}^{i}\left(\mathbf{J}_{l}^{i}\right), \\
\text { on ES } l^{+}, \\
\frac{1}{\eta_{0}} \mathbf{M}_{l}^{i}=-\left[\mathscr{L}_{l l}\right]^{-1} \cdot\left[-\frac{1}{2} \mathscr{J}_{l l}^{r}+\mathscr{K}_{l l}\right]\left(\mathbf{J}_{l}^{i}\right)=\mathscr{Z}_{H}^{i}\left(\mathbf{J}_{l}^{i}\right),
\end{array}
$$

on $\operatorname{ES} l^{+}$ 


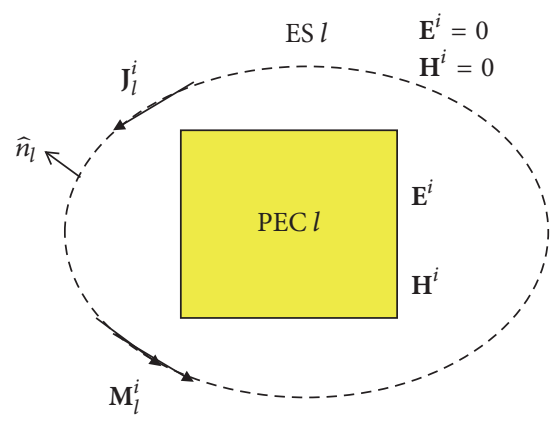

(a)

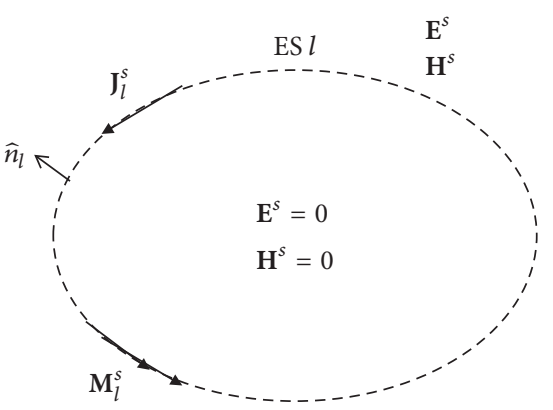

(b)

Figure 3: (a) Outside-in equivalence process. (b) Inside-out equivalence process.

Similarly, the relation between equivalence scattering currents $\mathbf{J}_{l}^{s}$ and $\mathbf{M}_{l}^{s}$ can be established via extinction theorem as shown in Figure 3(b):

$$
\begin{aligned}
& \frac{1}{\eta_{0}} \mathbf{M}_{l}^{s}=\left[\frac{1}{2} \mathscr{F}_{l l}^{r}+\mathscr{K}_{l l}\right]^{-1} \cdot \mathscr{L}_{l l}\left(\mathbf{J}_{l}^{s}\right)=\mathscr{Z}_{E}^{s}\left(\mathbf{J}_{l}^{s}\right), \\
& \text { on ES } l^{-}, \\
& \frac{1}{\eta_{0}} \mathbf{M}_{l}^{s}=-\left[\mathscr{L}_{l l}\right]^{-1} \cdot\left[\frac{1}{2} \mathscr{J}_{l l}^{r}+\mathscr{K}_{l l}\right]\left(\mathbf{J}_{l}^{s}\right)=\mathscr{X}_{H}^{s}\left(\mathbf{J}_{l}^{s}\right),
\end{aligned}
$$

on $\mathrm{ES} l^{-}$,

where ES $l^{-}$denotes the inside surface of ES $l$. It can be seen that there are two types of expressions for the magnetic current and electric current, which are named as E-type and $H$-type. Therefore, the scattering operator of single-source EPA on ES $l$ also has two types and can be written as

$$
\begin{aligned}
\mathbf{J}_{l}^{s}= & {\left[\mathscr{I}_{l l}\right]^{-1} \cdot\left[\widehat{n}_{l} \times \mathscr{K}_{l p}\right] \cdot\left[\eta_{0} \mathscr{L}_{p p}\right]^{-1} } \\
& \cdot\left[\eta_{0} \mathscr{L}_{p l}-\eta_{0} \mathscr{K}_{p l}\right] \cdot\left[\begin{array}{c}
1 \\
\mathscr{X}_{E / H}^{i}
\end{array}\right]\left(\mathbf{J}_{l}^{i}\right) \\
= & \widetilde{\mathcal{S}}_{l l}^{E / H} \cdot\left(\mathbf{J}_{l}^{i}\right) .
\end{aligned}
$$

The definition of scattering operator on ES $k$ is similar to the above. The two types of translation operator from ES $k$ to ES $l$ are defined as

$$
\widetilde{\mathscr{T}}_{l k}^{E / H}=\left[\mathscr{I}_{l l}\right]^{-1} \cdot\left[-\widehat{n}_{l} \times \mathscr{K}_{l k}-\widehat{n}_{l} \times \mathscr{L}_{l k}\right] \cdot\left[\begin{array}{c}
1 \\
\mathscr{Z}_{E / H}^{s}
\end{array}\right] .
$$

Finally, the single-source EPA equations have two types of expressions as follows:

$$
\left[\begin{array}{cccc}
\mathscr{I} & -\widetilde{\mathcal{S}}_{11}^{E / H} \widetilde{\mathscr{T}}_{12}^{E / H} & \cdots & -\widetilde{\mathcal{S}}_{11}^{E / H} \widetilde{\widetilde{T}}_{1 N}^{E / H} \\
-\widetilde{\mathcal{S}}_{22}^{E / H} \widetilde{\mathscr{T}}_{21}^{E / H} & \mathscr{I} & \cdots & -\widetilde{\mathcal{S}}_{22}^{E / H} \widetilde{\widetilde{T}}_{2 N}^{E / H} \\
\vdots & \vdots & \ddots & \vdots \\
-\widetilde{\mathcal{S}}_{N N}^{E / H} \widetilde{\mathscr{T}}_{N 1}^{E / H} & -\widetilde{\mathcal{S}}_{N N}^{E / H} \widetilde{\mathscr{T}}_{N 2}^{E / H} & \cdots & \mathscr{I}
\end{array}\right]
$$

$$
\cdot\left[\begin{array}{c}
\mathbf{J}_{1}^{s} \\
\mathbf{J}_{2}^{s} \\
\vdots \\
\mathbf{J}_{N}^{s}
\end{array}\right]=\left[\begin{array}{c}
\widetilde{\mathcal{S}}_{11}^{E / H} \cdot \mathbf{J}_{1}^{i} \\
\widetilde{\mathcal{S}}_{22}^{E / H} \cdot \mathbf{J}_{2}^{i} \\
\vdots \\
\widetilde{\mathcal{S}}_{N N}^{E / H} \cdot \mathbf{J}_{N}^{i}
\end{array}\right]
$$

Once the electric currents have been solved, the magnetic currents can be obtained using (15) or (16).

\section{Single-Source Tangential Equivalence Principle Algorithm}

In the single-source EPA, the equivalence surface currents $\mathbf{J}$ are the rotated tangential projections of the fields onto the surface as follows:

$$
\mathscr{I}\left(\mathbf{J}^{s / i}\right)= \pm \widehat{n} \times \mathbf{H}^{s / i}
$$

where $\mathbf{H}^{s / i}$ is the known field. Therefore both scattering operator and translation operator have identity operator in (17) and (18). Numerical results have shown that the discretization of identity operator using conventional Galerkin's scheme and common divergence-conforming RWG function leads to large error in the field projection procedure [10]. So the accuracy of single-source EPA needs to be improved. In this paper, a different field projection scheme is adopted to improve the accuracy by using surface integral to represent the tangential fields. As shown in Figures 3(a) and 3(b), the equivalent currents are generated from the tangential field projection on the equivalence surface ES $l$ by using Huygens equivalence principle as follows:

$$
\begin{aligned}
\eta_{0} \mathscr{L}_{l l}\left(\mathbf{J}_{l}^{i}\right)-\frac{1}{2} \mathscr{I}_{l l}^{r}\left(\mathbf{M}_{l}^{i}\right)-\mathscr{K}_{l l}\left(\mathbf{M}_{l}^{i}\right) & =\mathbf{E}^{i}, \quad \text { on } \mathrm{ES} l^{-}, \\
\frac{1}{2} \mathscr{I}_{l l}^{r}\left(\mathbf{J}_{l}^{i}\right)+\mathscr{K}_{l l}\left(\mathbf{J}_{l}^{i}\right)+\frac{1}{\eta_{0}} \mathscr{L}_{l l}\left(\mathbf{M}_{l}^{i}\right) & =\mathbf{H}^{i},
\end{aligned}
$$




$$
\begin{array}{cc}
\eta_{0} \mathscr{L}_{l l}\left(\mathbf{J}_{l}^{s}\right)+\frac{1}{2} \mathscr{J}_{l l}^{r}\left(\mathbf{M}_{l}^{s}\right)-\mathscr{K}_{l l}\left(\mathbf{M}_{l}^{s}\right)=\mathbf{E}^{s}, & \\
& \text { on ES } l^{+}, \\
-\frac{1}{2} \mathscr{J}_{l l}^{r}\left(\mathbf{J}_{l}^{s}\right)+\mathscr{K}_{l l}\left(\mathbf{J}_{l}^{s}\right)+\frac{1}{\eta_{0}} \mathscr{L}_{l l}\left(\mathbf{M}_{l}^{s}\right)=\mathbf{H}^{s}, & \mathrm{ES} l^{+} .
\end{array}
$$

By submitting (13) and (14) into (21) and (22) and submitting (15) and (16) into (23) and (24), there are four types of expressions for the incident current $\mathbf{J}_{l l}^{i}$ and scattering current $\mathbf{J}_{l l}^{s}$ separately, which can be denoted as TEE, TEH, THE, and $T H H$. Numerical results have shown that $\mathscr{X}_{H}^{i}$ and $\mathscr{Z}_{H}^{s}$ are more accurate than $\mathscr{Z}_{E}^{i}$ and $\mathscr{Z}_{E}^{s}$ when using RWG function as basis and testing functions [15]. It also has been proved that the THH type is the same as single-source EPA [16]. Therefore, only the TEH is used in this paper.

The single-source tangential EPA (T-EPA) based on the TEH type can be derived by submitting (14) into (21) and (16) into (23) as follows:

$$
\begin{aligned}
& \eta_{0} \mathscr{L}_{l l}\left(\mathrm{~J}_{l}^{i}\right)-\frac{1}{2} \eta_{0} \mathscr{F}_{l l}^{r} \cdot \mathscr{E}_{H}^{i}\left(\mathbf{J}_{l}^{i}\right)-\eta_{0} \mathscr{K}_{l l} \cdot \mathscr{X}_{H}^{i}\left(\mathbf{J}_{l}^{i}\right) \\
& \quad=\mathscr{X}_{T E H}^{i}\left(\mathbf{J}_{l}^{i}\right)=\mathbf{E}^{i}, \quad \text { on ES } l^{-}, \\
& \eta_{0} \mathscr{L}_{l l}\left(\mathbf{J}_{l}^{s}\right)+\frac{1}{2} \eta_{0} \mathscr{J}_{l l}^{r} \cdot \mathscr{L}_{H}^{s}\left(\mathbf{J}_{l}^{s}\right)-\eta_{0} \mathscr{K}_{l l} \cdot \mathscr{X}_{H}^{s}\left(\mathbf{J}_{l}^{s}\right) \\
& =\mathscr{Z}_{T E H}^{s}\left(\mathbf{J}_{l}^{s}\right)=\mathbf{E}^{s}, \quad \text { on ES } l^{+} .
\end{aligned}
$$

Then the scattering operator of single-source T-EPA on ES $l$ can be written as

$$
\begin{aligned}
\mathbf{J}_{l}^{s}= & {\left[\mathscr{Z}_{T E H}^{s}\right]^{-1} \cdot\left[\eta_{0} \mathscr{L}_{l p}\right] \cdot\left[\eta_{0} \mathscr{L}_{p p}\right]^{-1} } \\
& \cdot\left[\eta_{0} \mathscr{L}_{p l}-\eta_{0} \mathscr{K}_{p l}\right] \cdot\left[\begin{array}{c}
1 \\
\mathscr{Z}_{H}^{i}
\end{array}\right] \cdot \mathbf{J}_{l}^{i}=\widetilde{\mathcal{S}}_{l l}^{T E H} \cdot \mathbf{J}_{l}^{i}
\end{aligned}
$$

and the translation operator should be changed as

$$
\widetilde{\mathscr{T}}_{l k}^{T E H}=\left[\mathscr{Z}_{T E H}^{i}\right]^{-1} \cdot\left[\eta_{0} \mathscr{L}_{l k}-\eta_{0} \mathscr{K}_{l k}\right] \cdot\left[\begin{array}{c}
1 \\
\mathscr{Z}_{H}^{s}
\end{array}\right] .
$$

It can be seen that both of scattering operator and translation operator have the combination of $\mathscr{L}$ and $\pm(1 / 2) \mathscr{I}^{r}+$ $\mathscr{K}$ operators instead of using identity operator. So when using conventional Galerkin's scheme and RWG function to discretize the scattering and translation operators, this will lead to a more accuracy result [10]. Finally, the equations of single-source T-EPA are the same as (19) except for the expressions of scattering and translation operators.

\section{The Single-Source Tangential Equivalence Principle Algorithm with Source Reconstruction Method (SRM)}

In the practical engineering, the elements of array structures may be very close to each other. It is a challenge for EPA because the near fields between the equivalence surfaces

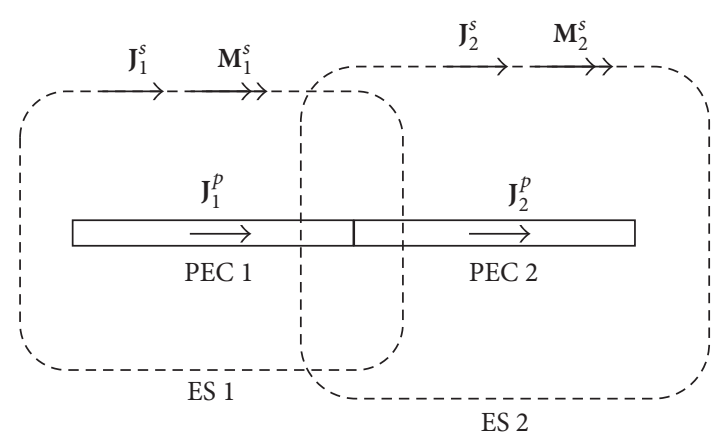

FIGURE 4: A PEC strip solved using source reconstruction method.

and objects are hard to calculate with high accurate. The other situation is that the elements are connected with each other, which can be solved with tap basis method. But this method needs to increase the number of unknowns and deteriorate the conditioning of EPA equations. In this paper, the source reconstruction method (SRM) is combined with single-source T-EPA to deal with the above two challenges. To illustrate this method, a scattering problem of a PEC strip is considered. The strip is divided into two parts as shown in Figure 4. The left part PEC 1 is enclosed by ES 1 , and the right part PEC 2 is enclosed by ES $2 . \mathbf{J}_{1}^{p}$ is the current on PEC $1 ; \mathbf{J}_{2}^{p}$ is the current on PEC 2. The MoM equations can be generated as

$$
\begin{aligned}
& \eta_{0} \mathscr{L}_{p_{1} p_{1}} \mathbf{J}_{1}^{p}+\eta_{0} \mathscr{L}_{p_{1} p_{2}} \mathbf{J}_{2}^{p}=-\mathbf{E}_{1}^{i}, \\
& \eta_{0} \mathscr{L}_{p_{2} p_{2}} \mathbf{J}_{2}^{p}+\eta_{0} \mathscr{L}_{p_{2} p_{1}} \mathbf{J}_{1}^{p}=-\mathbf{E}_{2}^{i} .
\end{aligned}
$$

The equivalence scattering currents $J_{1}^{s}$ on ES 1 can be obtained through inside-out propagation:

$$
\mathbf{J}_{1}^{s}=\left[\mathscr{E}_{T E H 1}^{\mathcal{s}}\right]^{-1} \cdot\left[\eta_{0} \mathscr{L}_{s_{1} p_{1}}\right] \cdot \mathbf{J}_{1}^{p},
$$

where the operator $\mathscr{Z}_{T E H 1}^{s}$ has been defined in (26). Then substituting (29) into (31) yields

$$
\begin{aligned}
\mathbf{J}_{1}^{s}= & {\left[\mathscr{E}_{T E H 1}^{s}\right]^{-1} \cdot\left[\eta_{0} \mathscr{L}_{s_{1} p_{1}}\right] \cdot\left[\eta_{0} \mathscr{L}_{p_{1} p_{1}}\right]^{-1} } \\
& \cdot\left(-\left[\eta_{0} \mathscr{L}_{p_{1} p_{2}}\right] \cdot \mathbf{J}_{2}^{p}-\mathbf{E}_{1}^{i}\right) .
\end{aligned}
$$

$\mathrm{E}_{1}^{i}$ is the incident plane wave on PEC 1 which is already known, while $\mathbf{J}_{2}^{p}$ needs to be solved. Suppose that the equivalence scattering currents $\mathbf{J}_{2}^{s}$ and $\mathbf{M}_{2}^{s}$ on ES 2 are already known, then the $\mathbf{J}_{2}^{p}$ can be got by using source reconstruction method [17] as follows:

$$
\mathbf{J}_{2}^{p}=\left[\eta_{0} \mathscr{L}_{s_{2} p_{2}}\right]^{+} \cdot\left[\mathscr{Z}_{T E H 2}^{s}\right] \cdot \mathbf{J}_{2}^{s},
$$

where $(\cdot)^{+}$denotes the pseudoinverse. In this paper, the pseudoinverse is computed by truncated SVD method. $\mathbf{E}_{1}^{i}$ can be replaced by equivalence incident currents as

$$
\mathbf{E}_{1}^{i}=\left[\eta_{0} \mathscr{L}_{p_{1} s_{1}}-\eta_{0} \mathscr{K}_{p_{1} s_{1}}\right] \cdot\left[\begin{array}{c}
1 \\
\mathscr{E}_{H 1}^{i}
\end{array}\right] \cdot \mathbf{J}_{1}^{i} .
$$


Hence, substituting (33) and (34) into (32), we have

$$
\mathbf{J}_{1}^{s}=\widetilde{\mathscr{T}}_{s_{1} s_{2}}^{\mathrm{SRM}} \cdot \mathbf{J}_{2}^{s}+\widetilde{\mathcal{S}}_{s_{1} s_{1}}^{T E H} \cdot \mathbf{J}_{1}^{i},
$$

where

$$
\begin{aligned}
\widetilde{\mathscr{T}}_{s_{1} s_{2}}^{\mathrm{SRM}}= & {\left[\mathscr{Z}_{\mathrm{TEH} 1}^{s}\right]^{-1} \cdot\left[\eta_{0} \mathscr{L}_{s_{1} p_{1}}\right] \cdot\left[\eta_{0} \mathscr{L}_{p_{1} p_{1}}\right]^{-1} } \\
& \cdot\left[-\eta_{0} \mathscr{L}_{p_{1} p_{2}}\right] \cdot\left[\eta_{0} \mathscr{L}_{s_{2} p_{2}}\right]^{+} \cdot\left[\mathscr{Z}_{\mathrm{TEH} 2}^{s}\right]
\end{aligned}
$$

and the definition of $\widetilde{\mathcal{S}}_{s_{1} s_{1}}^{T E H}$ is the same as (17). Similarly, the equation for the equivalence scattering currents on ES 2 can be written as

$$
\mathbf{J}_{2}^{s}=\widetilde{\mathscr{T}}_{s_{2} s_{1}}^{\mathrm{SRM}} \cdot \mathbf{J}_{1}^{s}+\widetilde{\mathcal{S}}_{s_{2} s_{2}}^{T E H} \cdot \mathbf{J}_{2}^{i} .
$$

Finally, the new EPA equations for connected structures can be obtained by combining (35) and (37):

$$
\left[\begin{array}{cc}
\mathscr{I} & -\widetilde{\mathscr{T}}_{s_{1} s_{2}}^{\mathrm{SRM}} \\
-\widetilde{\mathscr{T}}_{s_{2} s_{1}}^{\mathrm{SRM}} & \mathscr{I}
\end{array}\right] \cdot\left[\begin{array}{l}
\mathbf{J}_{1}^{s} \\
\mathbf{J}_{2}^{s}
\end{array}\right]=\left[\begin{array}{c}
\widetilde{\mathcal{S}}_{s_{1} s_{1}}^{T E H} \cdot \mathbf{J}_{1}^{i} \\
\widetilde{\mathcal{S}}_{s_{2} s_{2}}^{T E H} \cdot \mathbf{J}_{2}^{i}
\end{array}\right] .
$$

The coupling between PEC 1 and PEC 2 is calculated directly and there is no need to use buffer region. So the final unknowns are only equivalence surface currents by using the source reconstruction method. Equation (38) is simple and well-conditioned, which is similar to original EPA equation (10).

\section{Generalized Single-Source Tangential Equivalence Principle Algorithm}

By combing the above single-source EPA, tangential field projection, and source reconstruction method, the generalized single-source tangential equivalence principle algorithm (GSST-EPA) can be derived. Considering the scattering of $N$ connected objects, each object is enclosed by an equivalence surface as shown in Figure 5. Then the GSST-EPA equation can be written as

$$
\begin{gathered}
{\left[\begin{array}{cccc}
\mathscr{I} & -\mathscr{Z}_{12} & \cdots & -\mathscr{Z}_{1 N} \\
-\mathscr{Z}_{21} & \mathscr{I} & \cdots & -\mathscr{Z}_{2 N} \\
\vdots & \vdots & \ddots & \vdots \\
-\mathscr{Z}_{N 1} & -\mathscr{Z}_{N 2} & \cdots & \mathscr{I}
\end{array}\right] \cdot\left[\begin{array}{c}
\mathbf{J}_{1}^{s} \\
\mathbf{J}_{2}^{s} \\
\vdots \\
\mathbf{J}_{N}^{s}
\end{array}\right]} \\
=\left[\begin{array}{c}
\widetilde{\mathcal{S}}_{11}^{T E H} \cdot \mathbf{J}_{1}^{i} \\
\widetilde{\mathcal{S}}_{22}^{T E H} \cdot \mathbf{J}_{2}^{i} \\
\vdots \\
\widetilde{\mathcal{S}}_{N N}^{T E H} \cdot \mathbf{J}_{N}^{i}
\end{array}\right],
\end{gathered}
$$

where

$$
\mathscr{Z}_{i j}= \begin{cases}\widetilde{\mathcal{S}}_{i i}^{T E H} \widetilde{\mathscr{T}}_{i j}^{T E H}, & \text { if } i \text { and } j \text { are non-adjacent, } \\ \widetilde{\mathscr{T}}_{i j}^{\mathrm{SRM}}, & \text { if } i \text { and } j \text { are adjacent. }\end{cases}
$$

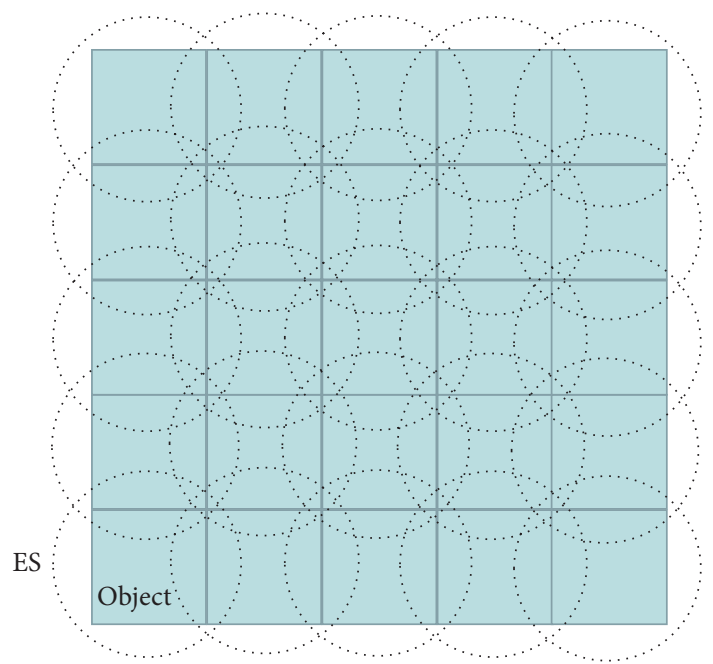

FIGURE 5: Each object is enclosed by an equivalence surface.

It should be pointed out that although the GSST-EPA can be used to solve generalized object, the GSST-EPA is much more suited for array structures because $\widetilde{\mathcal{S}}_{i i}^{T E H}$ is identical when the array element and equivalence surface are the same as each other. For the periodic array structures, $\widetilde{\mathscr{T}}_{i j}^{T E H}$ and $\widetilde{\mathscr{T}}_{i j}^{\text {SRM }}$ have the property of translation invariance which can be used to reduce the computing time and memory requirement. Therefore, the GSST-EPA is used to solve array structures in this paper.

To improve the ability of GSST-EPA to solve largescale array structures, multilevel fast multipole algorithm (MLFMA) is applied in GSST-EPA. This is because the calculating of mutual coupling between two equivalence surfaces is divided into two cases: adjacent case and nonadjacent case; then the MLFMA in GSST-EPA is different from that in original EPA. Firstly, the adjacent case is the near interaction actually which is solved by SRM. So the matrix $\left[\widetilde{\mathscr{T}}_{i j}^{\text {SRM }}\right]$ is calculated directly and stored. Secondly, the nonadjacent case is the far interaction which is solved by translation operator. So the translation matrix $\left[\widetilde{\mathscr{T}}_{i j}^{T E H}\right]$ is implemented using MLFMA instead of MoM.

\section{Numerical Results}

In this part, several numerical examples are given to show the accuracy and efficiency of the GSST-EPA.

The first example is to demonstrate the accuracy of GSST-EPA. The scattering of two PEC spheres in free space is investigated. The radius of PEC sphere is $0.3 \mathrm{~m}$. The distance between the centers of two spheres is $1.0 \mathrm{~m}$ in $x$-axis. Each sphere is enclosed by equivalence surface separately, which is a rectangle surface with $0.8 \times 0.8 \times 0.8 \mathrm{~m}^{3}$ as shown in Figure 6. The excitation is a polarized plane wave propagating into the negative direction at $300 \mathrm{MHz}$. The curvilinear Rao-Wilton-Glisson (CRWG) function [19] is used to discretize the spheres and equivalence surfaces. This case is solved by three kinds of methods separately: MoM, 


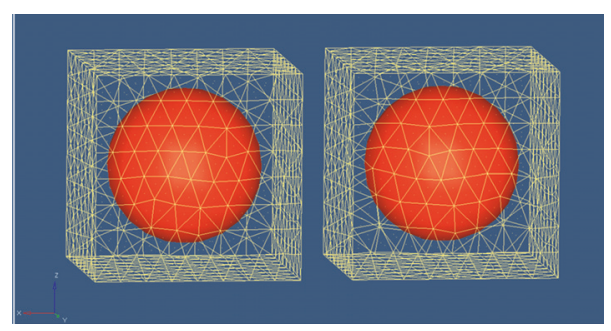

FIGURE 6: Two spheres are enclosed by two equivalence surfaces.

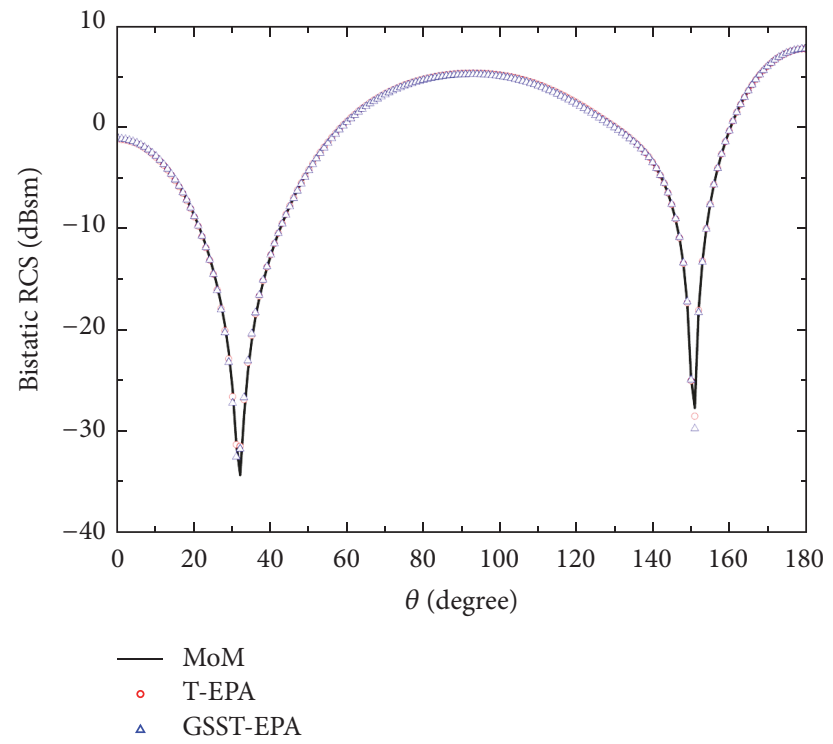

FIgure 7: The bistatic RCS of two PEC spheres with center distance $1.0 \mathrm{~m}$.

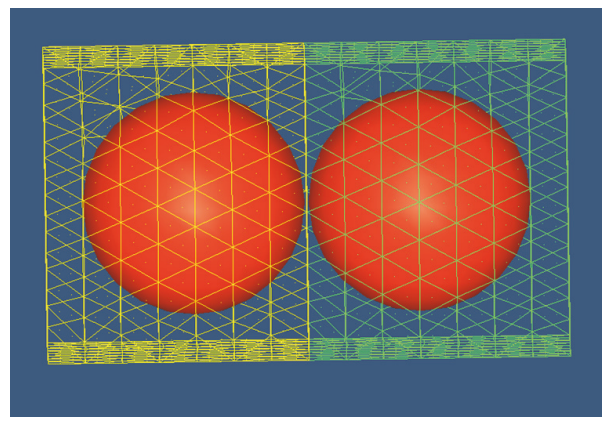

(a)

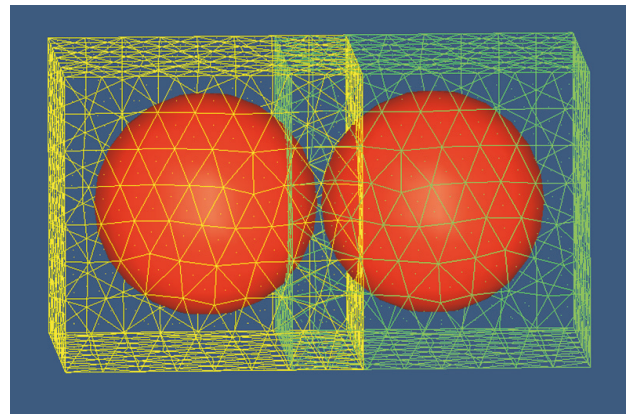

(b)

FIgure 8: Two PEC spheres enclosed by two ES, (a) T-EPA scheme, and (b) GSST-EPA scheme.

T-EPA [10], and GSST-EPA. The bistatic Radar Cross Section (RCS) results for the HH polarization are shown in Figure 7. It can be seen that the results agree with each other very well.

In the second example, the scattering of two PEC spheres is still investigated but changing the center distance from $1.0 \mathrm{~m}$ to $0.605 \mathrm{~m}$ as shown in Figure 8. In this case, it is difficult for the T-EPA scheme [10] to solve this problem accurately, because these spheres are too close with each other as shown in Figure 8(a). Then the equivalence surfaces are very close to the spheres which makes the field hard to calculate with good accurate. However, it is easy for the GSST-EPA to solve this case because the equivalence surfaces can intersect with each other as shown in Figure 8(b). In Figure 9, the bistatic RCS for the $\mathrm{HH}$ polarization of these two methods are compared with MoM. Obviously, the T-EPA result is incorrect while the GSST-EPA has good accuracy.

The third example shows a $5 \times 5$ sphere array solved by the new scheme. Each element is a dielectric-coated PEC sphere. The radius of PEC sphere is $0.2 \mathrm{~m}$. The thickness of the dielectric coating is $0.1 \mathrm{~m}$ with $\varepsilon_{r}=2.2, \mu_{r}=1.0$. The center 


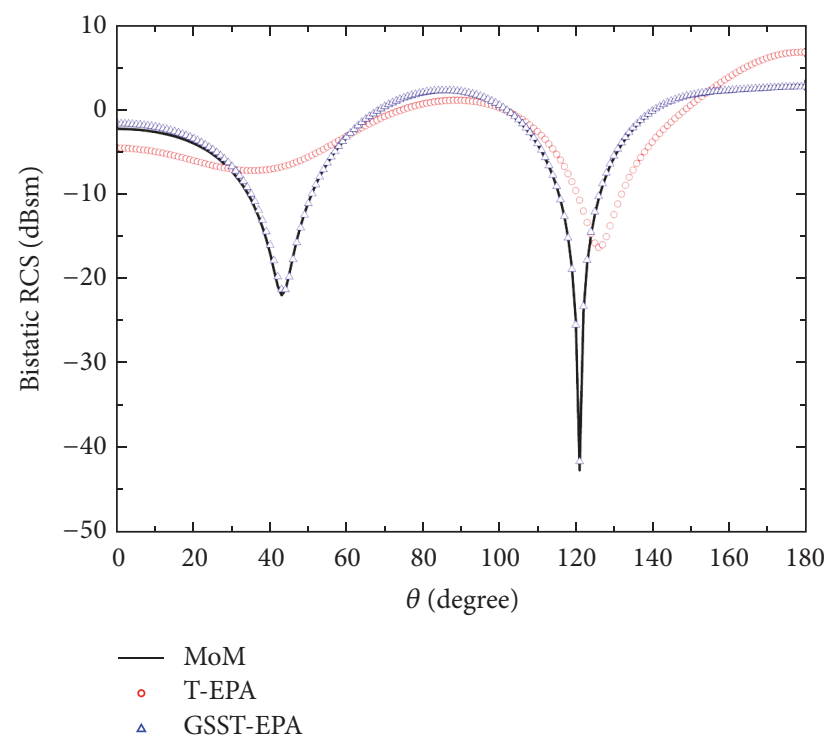

FIgure 9: The bistatic RCS of two PEC spheres with center distance $0.605 \mathrm{~m}$.

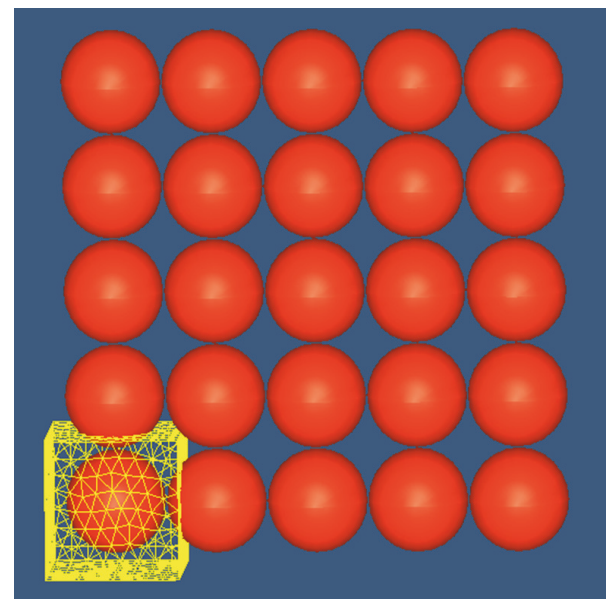

FIgURE 10: The model of a $5 \times 5$ sphere array.

distance between two spheres is $0.605 \mathrm{~m}$ in $\hat{x}$ and $\hat{y}$ directions. Each sphere is enclosed by an equivalence surface with $0.8 \times$ $0.8 \times 0.8 \mathrm{~m}^{3}$ as shown in Figure 10. The excitation is a polarized plane wave propagating into the negative $\widehat{z}$ direction at $300 \mathrm{MHz}$. In the GSST-EPA, each sphere is solved by volume surface integral equation (VSIE), and MLFMA is also used to accelerate the solution [20]. The result of VSIE_MLFMA with block diagonal preconditioner (BDP) is given as the benchmark as shown in Figure 11. The computational results are shown in Table 1. Obviously, GSST-EPA is more efficient than VSIE_MLFMA for solving this array structure. It is necessary to mention that the memory requirement has not been reduced much although the number of unknowns has been reduced by about $60 \%$. Because the equivalence surfaces can intersect with each other, couplings between the adjacent equivalence surfaces are calculated and stored directly. Only the coupling between the nonadjacent equivalence surfaces

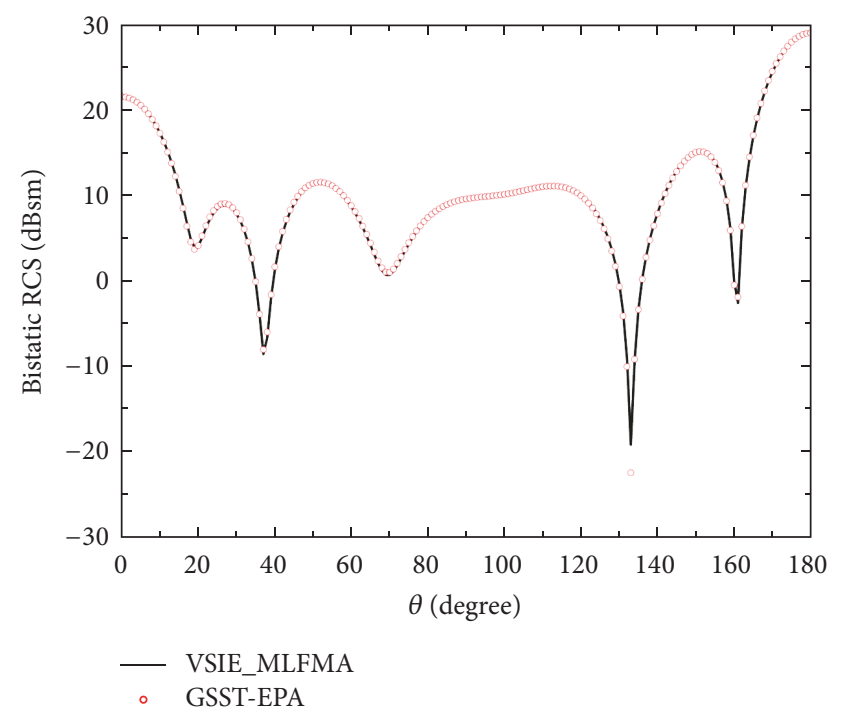

FIGURE 11: The bistatic RCS of a $5 \times 5$ sphere array; $\mathrm{HH}$ polarization at $300 \mathrm{MHz}$.

is calculated using MLFMA. Therefore the size of finest cube in MLFMA should be chosen twice as large as equivalence surface, which leads to the neighbor groups having a lot of unknowns.

Next, the scattering of a connected pyramid array is investigated. The dimension of a pyramid is shown in Figure 12(a) with $\varepsilon_{r}=2.0-j 0.04, \mu_{r}=2.0-j 0.04$. Each pyramid is enclosed by the same rectangle equivalence surface with $9 \times 9 \times 17 \mathrm{~cm}^{3}$. The distance between the centers of two elements is $7 \mathrm{~cm}$ which means that the elements connect with each other. The excitation is an $\hat{x}$ polarized plane wave propagating into the negative $\widehat{z}$ direction at $1.0 \mathrm{GHz}$. The array elements are solved by VIE. The comparison of bistatic $\mathrm{RCS}$ for $\mathrm{HH}$ polarization is shown in Figure 13. It can be seen that these two methods agree with each other very well. The computational results are shown in Table 2. Compared with VIE_MLFMA, the total solution time has been reduced by more than $90 \%$ in the GSST-EPA.

Finally, to demonstrate the efficiency and capability of the proposed method, the scattering of $10 \times 10$ pyramid array is solved. The parameters of the array are the same as that in the above example, except for the number of elements. The total number of unknowns of the array elements is 105,900 , while the number of unknowns on the equivalence surface is 23,400 . The error converging to 0.001 only needs 6 iteration steps. The total memory requirement is $3.2 \mathrm{~GB}$ and total solution time is 2,501 s. If the VIE_MLFMA is used to solve the array structure, then the memory requirement is more than $16 \mathrm{~GB}$. The result of bistatic RCS for the $\mathrm{HH}$ polarization is shown in Figure 14.

\section{Conclusions}

In this paper, a new scheme named GSST-EPA has been proposed to seek the scattering solution of array structures with very small distance or even connected array elements. 


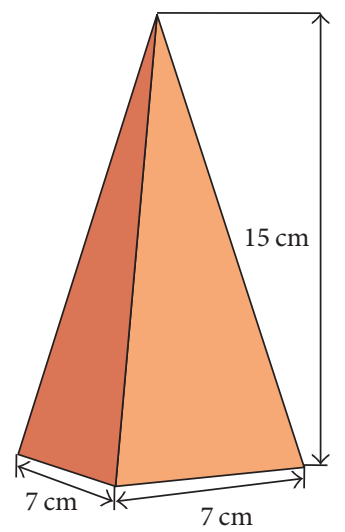

(a)

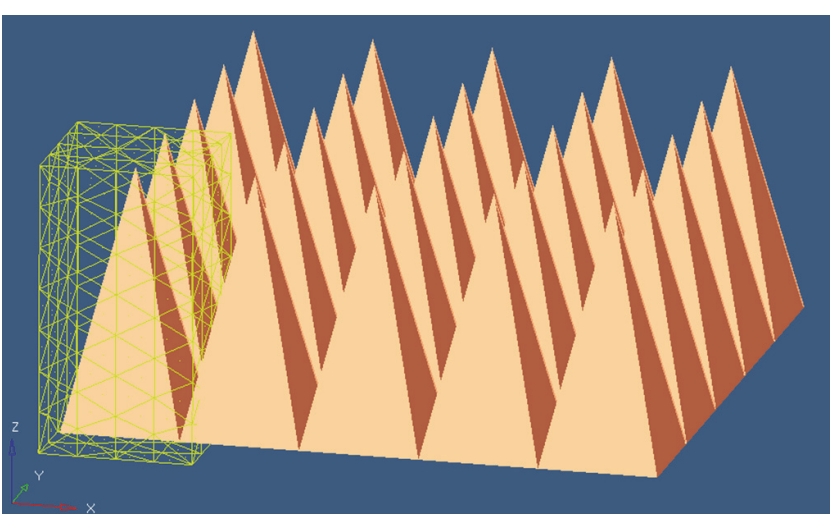

(b)

FIgURE 12: The model of pyramid array: (a) element; (b) $5 \times 5$ array and equivalence surface.

TABLE 1: The computational results of two methods.

\begin{tabular}{lcccc}
\hline Method & Unknowns & Memory $(\mathrm{MB})$ & Iteration number $(0.001)$ & Total time $(\mathrm{s})$ \\
\hline VSIE_MLFMA & 47,314 & $2,220.6$ & 251 & $4,442.4$ \\
GSST-EPA & 13,050 & $1,888.3$ & 22 & $2,421.3$ \\
\hline
\end{tabular}

TABLE 2: The comparison of computational results.

\begin{tabular}{lcccc}
\hline Method & Unknowns & Memory $(\mathrm{MB})$ & Iteration number $(0.001)$ & Total time $(\mathrm{s})$ \\
\hline VIE_MLFMA & 27,121 & $3,285.7$ & 11 & $8,112.7$ \\
GSST-EPA & 5,850 & 422.5 & 5 & 575.9 \\
\hline
\end{tabular}

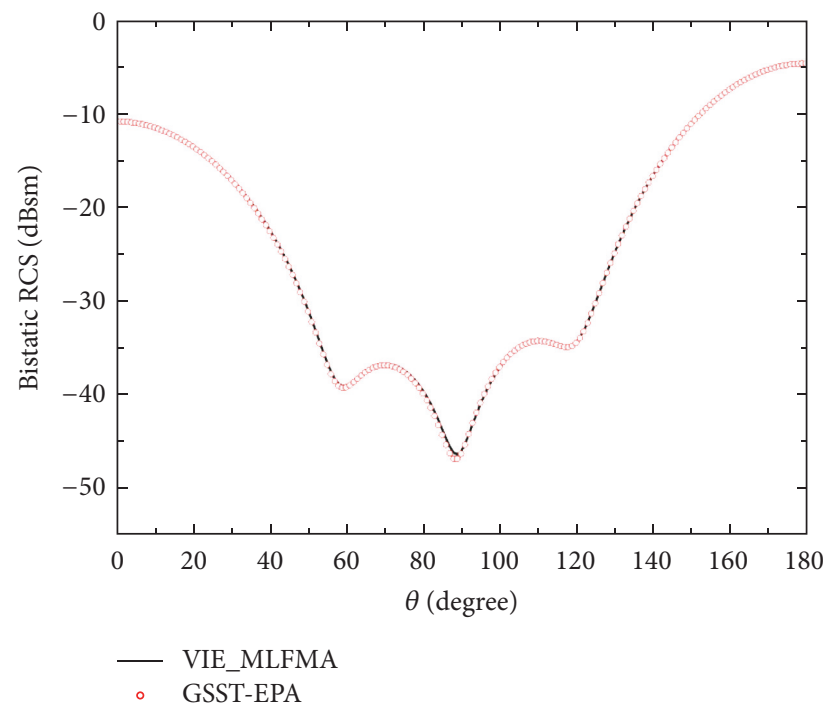

FIGURE 13: The bistatic RCS of $5 \times 5$ pyramid array; $\mathrm{HH}$ polarization at $1.0 \mathrm{GHz}$.

This scheme is based on the combination of single-source EPA, tangential field projection, and source reconstruction method. Compared with traditional EPA, this GSST-EPA can reduce the number of unknowns by half, improve the

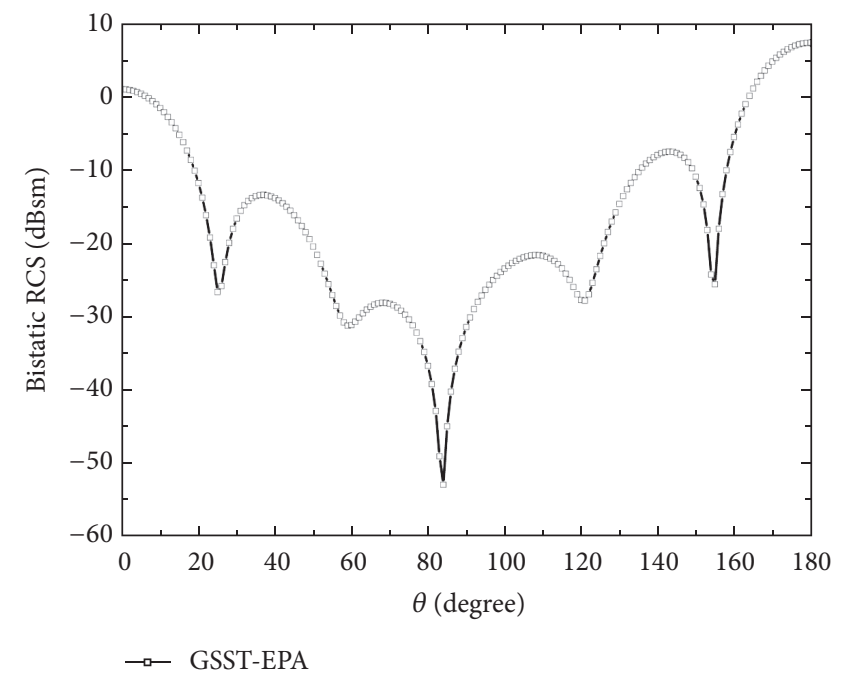

FIgURE 14: The bistatic RCS of $10 \times 10$ pyramid array; $\mathrm{HH}$ polarization at $1.0 \mathrm{GHz}$.

accuracy, and solve the connected structures. The MLFMA is applied further to improve the ability of GSST-EPA to solve large array problems. Numerical results show that the proposed method can solve the connected structures efficiently. 


\section{Conflicts of Interest}

The authors declare that there are no conflicts of interest regarding the publication of this paper.

\section{Acknowledgments}

This work is supported by National Natural Science Foundation of China (Grant no. 61501267, no. 61631012, no. 61671257, and no. 61425010), Zhejiang Provincial Natural Science Foundation of China (Grant no. LQ16F010003), and Ningbo University Discipline Project (Grant nos. XKL14D2058 and XYL15008) and is partially sponsored by K. C. Wong Magna Fund in Ningbo University.

\section{References}

[1] W. C. Chew and L. J. Jiang, "Overview of large-scale computing: The past, the present, and the future," Proceedings of the IEEE, vol. 101, no. 2, pp. 227-241, 2013.

[2] W. B. Lu, T. J. Cui, Z. G. Qian, X. X. Yin, and W. Hong, "Accurate analysis of large-scale periodic structures using an efficient subentire-domain basis function method," IEEE Transactions on Antennas and Propagation, vol. 52, no. 11, pp. 3078-3085, 2004.

[3] V. V. S. Prakash and R. Mittra, "Characteristic basis function method: a new technique for efficient solution of method of moments matrix equations," Microwave and Optical Technology Letters, vol. 36, no. 2, pp. 95-100, 2003.

[4] L. Matekovits, V. A. Laza, and G. Vecchi, "Analysis of large complex structures with the synthetic-functions approach," IEEE Transactions on Antennas and Propagation, vol. 55, no. 9, pp. 2509-2521, 2007.

[5] M.-K. Li, W. C. Chew, and L. J. Jiang, "A domain decomposition scheme based on equivalence theorem," Microwave and Optical Technology Letters, vol. 48, no. 9, pp. 1853-1857, 2006.

[6] M. Li and W. C. Chew, "Wave-field interaction with complex structures using equivalence principle algorithm," IEEE Transactions on Antennas and Propagation, vol. 55, no. 1, pp. 130-138, 2007.

[7] M.-K. Li and W. C. Chew, "Multiscale simulation of complex structures using equivalence principle algorithm with highorder field point sampling scheme," Institute of Electrical and Electronics Engineers. Transactions on Antennas and Propagation, vol. 56, no. 8, part 1, pp. 2389-2397, 2008.

[8] A. M. Van De Water, B. P. De Hon, M. C. Van Beurden, A. G. Tijhuis, and P. De Maagt, "Linear embedding via Greenâs operators: A modeling technique for finite electromagnetic bandgap structures," Physical Review E - Statistical, Nonlinear, and Soft Matter Physics, vol. 72, no. 5, Article ID 056704, 2005.

[9] S. Xiang, G. Xiao, X. Tian, and J. Mao, "Analysis of large-scale phased antenna array with generalized transition matrix," IEEE Transactions on Antennas and Propagation, vol. 61, no. 11, pp. 5456-5464, 2013.

[10] P. Ylä-Oijala and M. Taskinen, "Electromagnetic scattering by large and complex structures with surface equivalence principle algorithm," Waves in Random and Complex Media, vol. 19, no. 1, pp. 105-125, 2009.

[11] H. Shao, J. Hu, H. Guo, F. Ye, W. Lu, and Z. Nie, "Fast simulation of array structures using T-EPA with hierarchical LU decomposition," IEEE Antennas and Wireless Propagation Letters, vol. 11, pp. 1560-1563, 2012.
[12] Z.-H. Ma, L. J. Jiang, and W. C. Chew, "Loop-tree free augmented equivalence principle algorithm for low-frequency problems," Microwave and Optical Technology Letters, vol. 55, no. 10, pp. 2475-2479, 2013.

[13] Y. Shi, J. Wang, and C.-H. Liang, "A time-domain equivalence principle and its marching-on-in-degree solution," Microwave and Optical Technology Letters, vol. 56, no. 10, pp. 2415-2422, 2014.

[14] T. Su, L. Du, and R. Chen, "Electromagnetic scattering for multiple PEC bodies of revolution using equivalence principle algorithm," Institute of Electrical and Electronics Engineers. Transactions on Antennas and Propagation, vol. 62, no. 5, pp. 2736-2744, 2014.

[15] H. Shao, J. Hu, and W. C. Chew, "Single-source equivalence principle algorithm for the analysis of complex structures," IEEE Antennas and Wireless Propagation Letters, vol. 13, pp. 12551258, 2014.

[16] H. Shao, J. Dong, and J. Hu, "Improving the Accuracy of SingleSource Equivalence Principle Algorithm by Using Tangential Field Projection Scheme," Electromagnetics, vol. 36, no. 8, pp. 534-545, 2016.

[17] H. Shao and J. Hu, "Analysis of connected structures using equivalence principle algorithm with source reconstruction method," Journal of Electromagnetic Waves and Applications, vol. 30, no. 13, pp. 1740-1754, 2016.

[18] J. M. Song and W. C. Chew, "Multilevel fast-multipole algorithm for solving combined field integral equations of electromagnetic scattering," Microwave and Optical Technology Letters, vol. 10, no. 1, pp. 14-19, 1995.

[19] S. Wandzura, "Electric current basis functions for curved surfaces," Electromagnetics, vol. 12, no. 1, pp. 77-91, 1992.

[20] C. C. Lu and W. C. Chew, "Electromagnetic scattering from material coated PEC objects: a hybrid volume and surface integral equation approach," in Proceedings of the IEEE Antennas and Propagation Society International Symposium, IEEE, pp. 2562-2565, Orlando, FL, USA, July, 1999. 


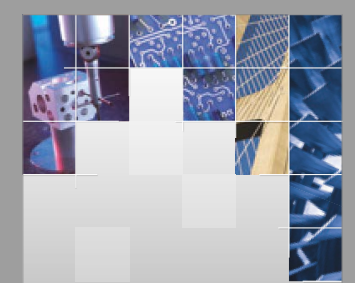

\section{Enfincering}
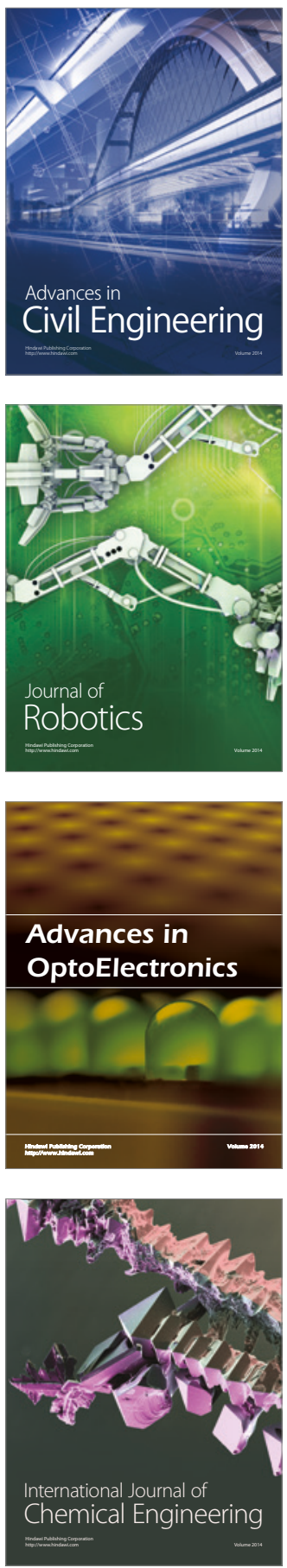

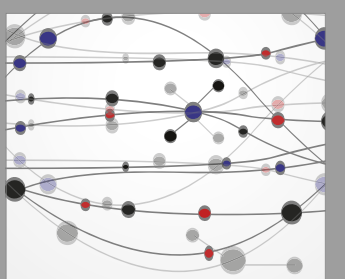

The Scientific World Journal

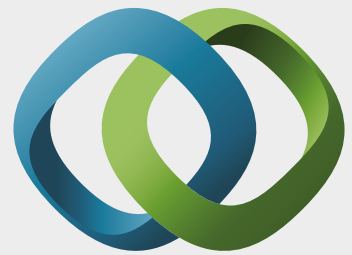

\section{Hindawi}

Submit your manuscripts at

https://www.hindawi.com
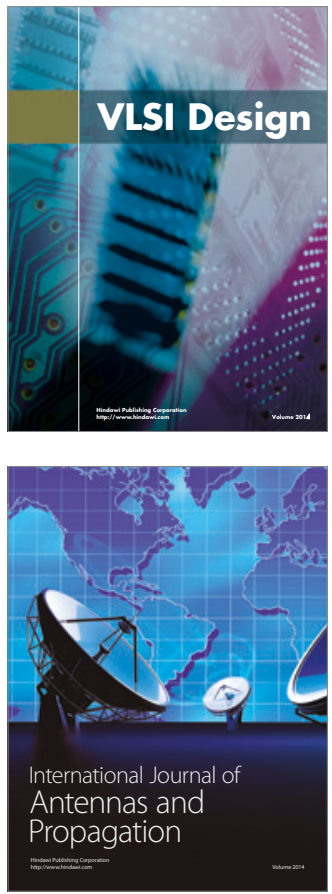

\section{Rotating}

Machinery
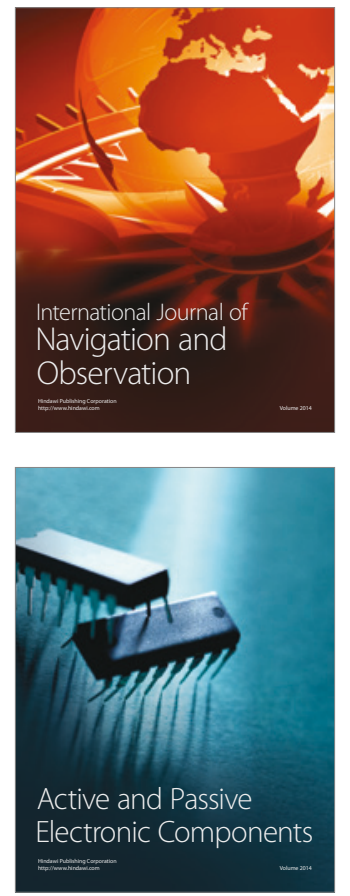
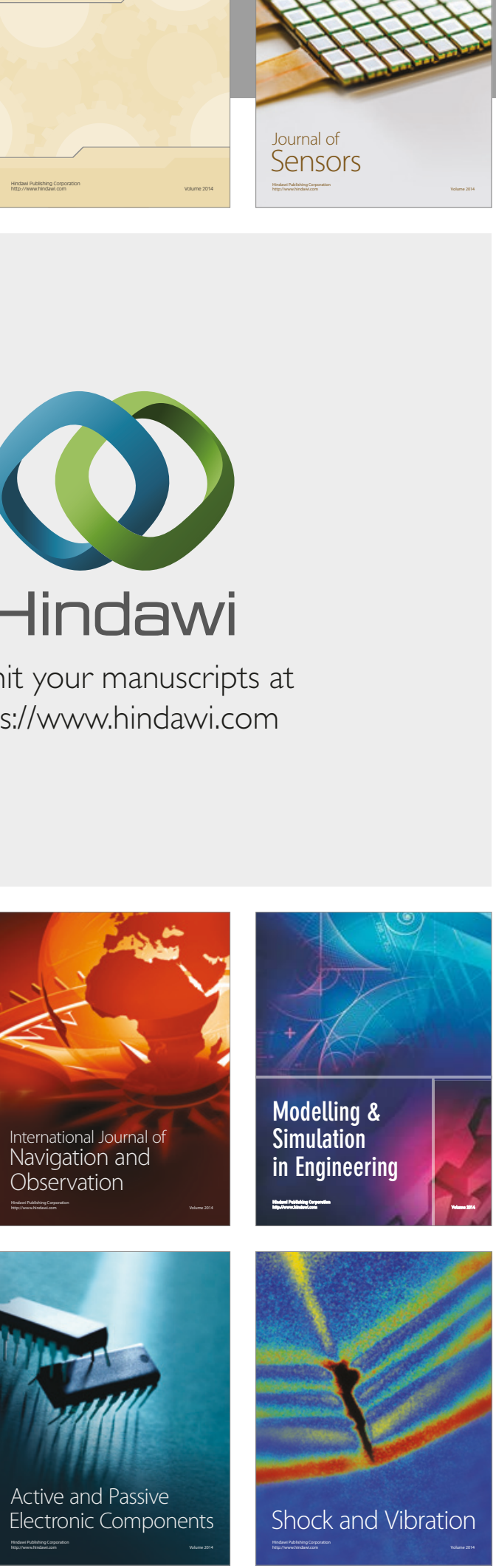
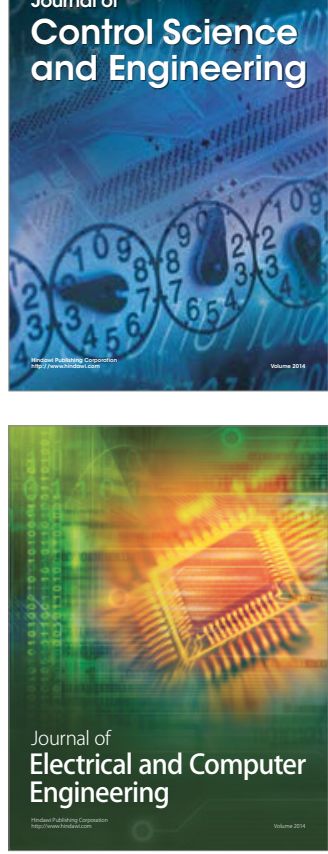

Distributed

Journal of

Control Science

and Engineering
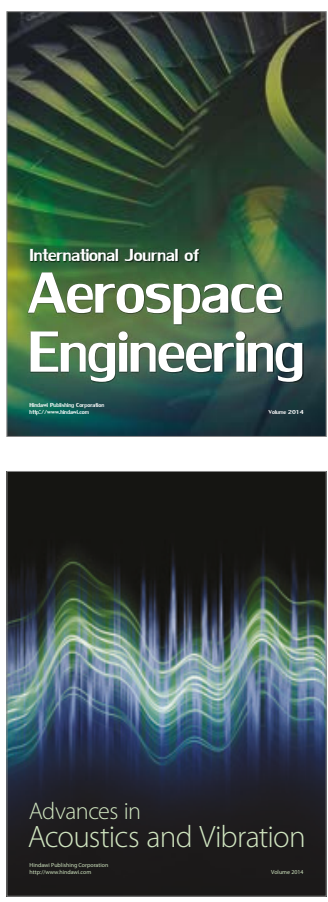

Sensor Networks 